\title{
The association between platelet indices and deep surgical site infection after open induction internal fixation for traumatic limb fractures
}

This article was published in the following Dove Press journal: Infection and Drug Resistance

\author{
Zitao Zhang' \\ Yuanhui Ji² \\ Zhen Wang' \\ Xusheng Qiu' \\ Yixin Chen' \\ 'Department of Orthopedics, The \\ Affiliated Drum Tower Hospital of \\ Nanjing University Medical School, \\ Nanjing 210008, China; ${ }^{2}$ Department \\ of Clinical Laboratory, The Affiliated \\ Drum Tower Hospital of Nanjing \\ University Medical School, Nanjing \\ 210008 , China
}

\begin{abstract}
Objectives: Deep surgical site infection (DSSI) is one of the most serious complications after open induction internal fixation (ORIF) for traumatic limb fractures. In this study, we aimed to investigate the diagnostic role of platelet indices (platelet count [PLT], mean platelet volume [MPV], and platelet distribution width [PDW]) in DSSI.

Patients and methods: Data obtained between January 2011 and December 2017 in The Affiliated Drum Tower Hospital of Nanjing University Medical School from cases ( $n=29)$ with DSSI and fracture control subjects $(n=29)$ matched for age, gender, and fracture type were analyzed. The white blood cell (WBC) count, neutrophil count, neutrophil percentage, and platelet indices from blood samples were compared between case and control groups. In addition, the cutoff value, sensitivity, and specificity were calculated by receiver-operating characteristic (ROC) curves.
\end{abstract}

Results: No significant differences were detected in demographic features, the WBC count, neutrophil count, neutrophil percentage, and MPV values between two groups $(P>0.05)$. The PLT values were significantly higher in the case group than in the control group (303.00 \pm 139.27 vs $\left.196.10 \pm 59.61\left[10^{9} / \mu \mathrm{L}\right], P=0.001\right)$. The PDW values of the case and control groups were $11.77 \pm 2.71$ and $13.19 \pm 2.39 \%$, respectively, and were significantly lower in the case group $(P=0.001)$. ROC curve analysis suggested a cutoff point for PLT as $215.50\left(10^{9} / \mu \mathrm{L}\right.$, larger values indicate pathology) for the diagnosis of DSSI with the sensitivity and specificity of 79.3 and $72.4 \%$, respectively. For PDW, the cutoff point was $10.35 \%$ (smaller values indicate patients) for the diagnosis of DSSI with the sensitivity and specificity of 37.9 and $96.6 \%$, respectively.

Conclusion: Our results suggest that PDW combined with PLT can be used as an important additional test for the diagnosis of DSSI after ORIF for traumatic limb fractures, thus reducing the cost and loss of time.

Keywords: surgical site infection, internal fracture fixation, fracture, platelet count, platelet function test

\section{Introduction}

Deep surgical site infection (DSSI) after open induction internal fixation (ORIF) is a serious complication for traumatic limb fracture surgery, and if not promptly diagnosed or managed, it often leads to catastrophic consequences such as amputation and even death. ${ }^{1}$ Although diagnostic examinations (including laboratory tests, imaging modalities, and pathogen cultures) exist, rapid, cheap, and accurate diagnosis remains challenging for many patients with infections. ${ }^{2}$ Complete blood count (CBC), one of the most common routine preoperative tests, is frequently used by surgeons to assess
Correspondence: Yixin Chen

Department of Orthopedics, The Affiliated Drum Tower Hospital of Nanjing University Medical School, 32I, Zhongshan Road, Nanjing 210008, China Tel/fax +862583I06666 Email chenyixin93@126.com 
inflammatory pathologies. Orthopedists often pay attention to white blood cell (WBC) count and neutrophil count in CBC to diagnose or monitor infections; however, their sensitivity and specificity are very low in fracture patients. ${ }^{2,3}$ Besides WBC count and neutrophil count, recent studies gradually focus on the role of platelet indices including platelet count (PLT), mean platelet volume (MPV), and platelet distribution width (PDW) in diagnosing infections such as acute appendicitis, ventriculo-peritoneal shunt infection, and urinary tract infection. ${ }^{4-7}$ To our knowledge, there are no studies conducted examining the diagnosis of DSSI after ORIF for traumatic limb fractures using platelet indices. In this study, we aim to determine whether PDW, PLT, and MPV could be used for the diagnosis of such DSSI.

\section{Patients and methods}

\section{Patients}

A case-control study was performed. Between January 2011 and December 2017, a total of 73 patients with infections after ORIF were admitted at the Department of Orthopedics, The Affiliated Drum Tower Hospital of Nanjing University Medical School. DSSI was classified when both of the following criteria were met at the same time: clinical signs of a surgical site infection and positive bacterial cultures taken from the secreting wound. ${ }^{8}$ Patients were excluded from the study for 1) age $\leq 18$ years; 2) antibiotics or other treatments given before; 3 ) having acute or chronic infectious disease, comorbid conditions (cardiac, respiratory, renal, endocrinal, and vascular disease, cancer, etc.), hematological disease and blood transfusion within the last year for any reason; 4) having other organs' injuries such as brain, liver, and spleen; and 5) using ongoing medication (analgesics, oral contraceptives, antimetabolites, etc.). As a result, 29 patients per group were enrolled in this study. The control group was matched for age ( \pm 3 years), gender (male/female: $26 / 3$ ), and fracture type (the same fracture type according to [Association for the Study of Internal Fixation; AO] classification) as closely as possible to patients with DSSI. The collection of patients' blood and all protocols or experiments were undertaken with the written informed consent of patients and with approval from the Ethics Committee of The Affiliated Drum Tower Hospital of Nanjing University Medical School. Our studies were conducted in compliance with the Declaration of Helsinki.

\section{Blood test}

All blood samples (1.5 mL for each patient) were obtained from a peripheral vein on the day of admission and stored in tubes containing EDTA and assayed automatically using internationally certified devices (XE-5000; Sysmex, Japan). The reference values in The Affiliated Drum Tower Hospital of Nanjing University Medical School laboratory were (3.5$9.5) \times 10^{9} / \mu \mathrm{L}$ for $\mathrm{WBC}$ count, $(1.8-6.3) \times 10^{9} / \mu \mathrm{L}$ for neutrophil count, $40-70 \%$ for neutrophil percentage, $(181-300) \times 10 \% / \mu \mathrm{L}$ for PLT, 9.4-12.5 fL for MPV, and 15.5-18.1\% for PDW. All results were approved by an independent biochemistry expert who was blind to the patients' histories. According to our clinical experiences, if the values of blood test were to high or low, the test would be repeated again to avoid measurement error.

\section{Statistical analyses}

Statistical analyses were performed, using the SPSS for Windows Version 18.0 (SPSS Inc., Chicago, IL, USA). Categorical variables are shown as frequencies and continuous variables were expressed as mean $\pm \mathrm{SD}$. The paired $t$-test was used for continuous variables. Chi-squared test was used to analyze categorical variables. To establish the predictive value of the parameters for diagnosing DSSI, receiver-operating characteristic (ROC) curves were analyzed. The areas under the ROC curves (AUC), the specificity and sensitivity were calculated and the optimal cutoff point from the study was the threshold leading to the maximum summation of sensitivity and specificity (the Youden index). ${ }^{9}$ All differences were considered significant at a value of $P<0.05$.

\section{Results}

Twenty-nine infected cases and matched-control patients were included in this study. The body mass index (case group: $23.0 \pm 3.7$ vs control group: $23.1 \pm 3.1 \mathrm{~kg} / \mathrm{m}^{2}$ ) and the smoking and drinking ratio distribution had no significant differences between two groups (Table $1, P>0.05$ ).

The comparisons of blood parameters between case and control group were given in Table 2 . No significant differences were either detected in the WBC count, neutrophil count, neutrophil percentage, or MPV values between the two groups $(P>0.05)$. There were statistical differences in PLT and PDW between two groups $(P<0.05)$. The case group had a mean PLT of 303.00 \pm 139.27 , and the control group had a mean PLT of $196.10 \pm 59.61\left(10^{9} / \mu \mathrm{L}\right)$. The PLT values were significantly higher in the case group than in the control group $(P=0.001)$. The PDW values of the case and control groups were $11.77 \pm 2.71$ and $13.19 \pm 2.39 \%$, respectively. The PDW values were significantly lower in the case group $(P=0.001)$.

For ROC analysis, using a cutoff value for PLT at 215.50 $\left(10^{9} / \mu \mathrm{L}\right)$, the sensitivity, specificity, and positive predictive 
value (PPV) were 79.3, 72.4, and 74.2\%; using a cutoff value for PDW at $10.35(\%)$, the sensitivity, specificity, and PPV was $37.9,96.6$, and $91.8 \%$ for the prediction of DSSI (Table $3)$. The PLT ROC analysis curves yielded an AUC of 0.812 (95\% CI: $0.700-0.924)$ in the prediction of developing DSSI (Figure 1A). For the PDW analysis, ROC curves yielded an AUC of 0.697 (95\% CI: 0.562-0.832) in predicting the development of DSSI (Figure 1B). At last, PLT $\left(215.50>10^{9} / \mu \mathrm{L}\right)$ and PDW $(<10.35 \%)$ were combined with logistic regressions to make ROC analysis. The sensitivity, specificity, PPV, and AUC were 72.4, 82.8, 80.8, and 0.817 (95\% CI: 0.704-0.930) (Table 3 and Figure 1C).

\section{Discussion}

DSSI is a dreaded complication after ORIF for traumatic limb fractures. Mild infection may delay fracture healing and prolong hospital stay, while severe infection often causes amputation or even death. ${ }^{1}$ Early detection of DSSI is the first step to prevent or decrease its damage. Although some diagnostic methods are used to help diagnose DSSI, complete blood cell test is still the easiest, cheapest, and fastest one. In this study, we first revealed that platelet indices including PLT $\left(>215.50 \times 10^{9} / \mu \mathrm{L}\right)$ and PDW $(<10.35 \%)$ could predict DSSI after ORIF for traumatic limb fractures.

WBC and neutrophil are the most commonly inflammatory marker, and their changes are usually used to indicate the onset of infectious disease. However, WBC and neutrophil are affected by many factors such as physiological stress, treatment, and trauma. ${ }^{3,8}$ Especially in fracture patients, the increase in WBC and neutrophil often appears. Considering DSSI is an inflammatory process, we also compared WBC and neutrophil parameters between case and control groups. Consistent with previous studies, there were no significant differences of WBC count, neutrophil count, and neutrophil percentage between two groups. ${ }^{2}$ Hence. it was not necessary to further make ROC analysis.

Table I The demographics between case and control groups

\begin{tabular}{|l|l|l|l|}
\hline & Cases $(\mathbf{n}=29)$ & Controls $(\mathbf{n}=29)$ & $P$-value \\
\hline BMI $\left(\mathrm{kg} / \mathrm{m}^{2}\right)^{\mathrm{a}}$ & $23.0 \pm 3.7$ & $23.1 \pm 3.1$ & 0.895 \\
Daily drinker (yes/no) & $8 / 21$ & $10 / 11$ & $0.232^{\mathrm{b}}$ \\
Current smoker (yes/no) & $5 / 24$ & $8 / 21$ & $0.530^{\mathrm{b}}$ \\
\hline
\end{tabular}

Notes: $P<0.05$ indicated significances. ${ }^{a}$ Values are presented as mean $\pm S D$. ' Fisher's exact test.

Abbreviation: BMI, body mass index.

Table 2 Comparison of blood parameters between case and control groups

\begin{tabular}{|c|c|c|c|c|c|}
\hline & $\begin{array}{l}\text { Case group } n=29, \\
\text { mean } \pm \text { SD }\end{array}$ & $\begin{array}{l}\text { Control group } n=29 \text {, } \\
\text { mean } \pm \text { SD }\end{array}$ & $\begin{array}{l}\text { Mean } \\
\text { difference }\end{array}$ & $\begin{array}{l}95 \% \mathrm{Cl} \text { of mean } \\
\text { difference }\end{array}$ & $P$-value \\
\hline WBC count $\left(10^{9} / \mu \mathrm{L}\right)$ & $7.72 \pm 2.79$ & $8.48 \pm 2.63$ & 0.75 & $-0.70-2.20$ & 0.298 \\
\hline Neutrophil count $\left(10^{9} / \mu \mathrm{L}\right)$ & $5.25 \pm 2.93$ & $5.90 \pm 2.49$ & 0.73 & $-0.84-2.14$ & 0.382 \\
\hline Neutrophil percentage & $65.16 \pm 11.37$ & $68.91 \pm 11.69$ & 3.75 & $-3.48-10.97$ & 0.297 \\
\hline MPV (fL) & $10.28 \pm 1.28$ & $10.81 \pm 1.11$ & 0.53 & $-0.079-1.15$ & 0.085 \\
\hline $\operatorname{PLT}\left(10^{\%} / \mu \mathrm{L}\right)$ & $303.00 \pm 139.27$ & $196.10 \pm 59.61$ & -106.90 & $-164.63--49.15$ & 0.001 \\
\hline PDW (\%) & $|1.77 \pm 2.7|$ & $13.19 \pm 2.39$ & 1.80 & $0.77-2.83$ & 0.001 \\
\hline
\end{tabular}

Note: $P<0.05$ indicated significant differences.

Abbreviations: MPV, mean platelet volume; PDW, platelet distribution width; PLT, platelet count; WBC, white blood cell.

Table 3 Results of ROC analysis for blood parameters

\begin{tabular}{|l|l|l|l|l|l|l|}
\hline & $\begin{array}{l}\text { Cutoff } \\
\text { point }\end{array}$ & Sensitivity (\%) & Specificity (\%) & AUC & PPV (\%) & $\begin{array}{l}\text { Asymptomatic 95\% CI } \\
\text { lower/upper bound }\end{array}$ \\
\hline PLT $\left(10^{\%} / \mu \mathrm{L}\right)$ & 215.50 & 79.3 & 72.4 & 0.812 & 74.2 & $0.700-0.924$ \\
PDW (\%) & 10.35 & 37.9 & 96.6 & 0.697 & 91.8 & $0.562-0.832$ \\
PLT + PDW & & 72.4 & 82.8 & 0.817 & 80.8 & $0.704-0.930$ \\
\hline
\end{tabular}

Abbreviations: AUC, areas under the ROC curves; PDW, platelet distribution width; PLT, platelet count; PPV, positive predictive value; ROC, receiver-operating characteristic. 

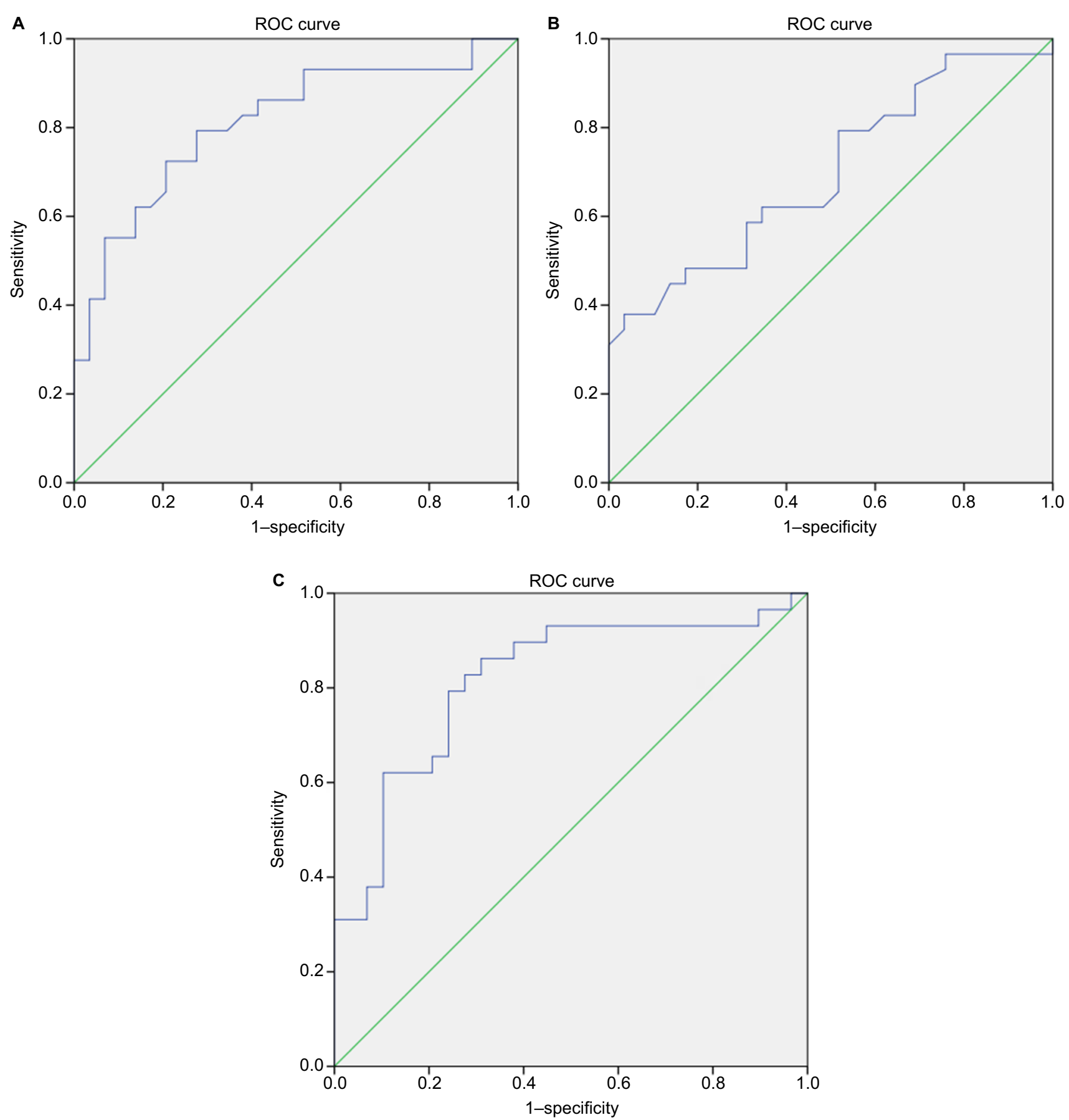

Figure I The ROC curves for the optimal cutoff value of platelet indices.

Notes: (A) The ROC curve for PLT (larger values indicate infection). (B) The ROC curve for PDW (smaller values indicate infection). (C) The ROC curve for PDW in combination with PLT.

Abbreviations: PDW, platelet distribution width; PLT, platelet count; ROC, receiver operating characteristic.

Platelets, the small, anucleate cell fragments circulating in blood, play a basic role in hemostasis and thrombosis. ${ }^{10}$ In recent years, platelets have been emphasized with respect to some infections and inflammatory diseases. ${ }^{4-7,10-12}$ Platelet indices including MPV, PLT, and PDW are the most common used parameters and can be easily examined in CBC. MPV represents the average size of platelets and correlates with platelet function and activation. ${ }^{13}$ Larger platelets are metabolically and enzymatically more active in inflammatory process. ${ }^{10} \mathrm{PDW}$, an index of platelet 
size heterogeneity, is also known as the volume change coefficient and represents heterogenic demarcation of megakaryocytes rather than the aging of circulating thrombocytes. ${ }^{7}$ Increased PDW levels indicate platelets swelling, destruction, and immaturity. ${ }^{13}$

In different infections or inflammatory diseases, platelet indices had different changes. For example, MPV levels were higher in urinary tract infection but lower in acute appendicitis. ${ }^{6,7}$ PDW levels were increased in acute appendicitis while reduced in invasive fungal disease. ${ }^{7,11}$ In the current study, we first found that there were significant correlations between platelet indices (PLT and PDW) with DSSI. PDW levels were reduced while PLT levels were increased in patients with DSSI $(P<0.05)$. However, there were no differences in MPV between two groups. We speculated that at the onset of DSSI, large platelets initiated to play an antibacterial role and be gradually reduced while megakaryocytes had not yet been stimulated to release large platelets from bone marrow. Thus, PDW seems to have been decreased in DSSI patients. In contrast, the infection could promote the inflammation phase interleukins releasing, such as interlecukin-1 and interlecukin- 6 , thus enhancing bone marrow activity. ${ }^{14}$ Thrombocytosis may occur after bone marrow activity stimulated, which may be the reason for PLT levels' increase in DSSI patients.

In the current trial, the values of sensitivity and specificity of PLT in our study, 79.3 and $72.4 \%$, respectively, are relatively moderate. Meanwhile, the best cutoff point of $10.35 \%$ for PDW in the diagnosis of DSSI had a low sensitivity of $37.9 \%$ but a high specificity of $96.6 \%$. Explanation for PDW's low sensitivity found in our study may be attributed to our strict inclusion criteria and small sample size. Due to the small sample size, the number of patients with PDW $<10.35 \%$ was 1 in the control group and 11 in the case group and the proportion of such patients was only $26.1 \%$ in the sample. Therefore, the low sensitivity $37.9 \%$ of PDW might cause by the rareness of PDW $<10.35 \%$ in this study. However, the PPV (91.8\% when PDW $<10.35 \%)$ was obviously higher than $74.2 \%$ when PLT $>215.5(10 \%$ $\mu \mathrm{L})$. Hence, the high PPV indicated that PDW could be very powerful to screen the true positives when its value was less than $10.35 \%$. To DSSI, high PPV was very important for its early and exact diagnosis could strongly affect the patient's treatment and prognosis. Now that using PLT or PDW alone for diagnosis both had some disadvantages, we then made a combined ROC analysis with PLT and PDW. PDW in combination with PLT could make all the statistical indices in adequate and range. It is acknowledged that the platelet indices cannot be the sole diagnostic tool for DSSI.
It must be very discreet to make such diagnosis, because once DSSI was diagnosed improperly, further treatments of DSSI such as large doses of antibiotics, debridement and hard removal might cause additional severe harm to patients. However, attempts to develop a diagnostic method with $100 \%$ specificity and sensitivity have failed so far.

Our study has some limitations: first, as an observational study, the diagnostic role of platelet indices in DSSI after ORIF requires to be investigated further in a prospective validation study. Second, due to the low morbidity rate of DSSI in our department, the number of patients was not many. Multicenter prospective studies with large sample size are necessary in the future.

\section{Conclusion}

Using the PLT and PDW simultaneously could make all the statistical indices in adequate range including specificity, sensitivity, PPV, and AUC. Hence, it was recommended that both PDW in combination with PLT should be used for the diagnosis of DSSI after ORIF for traumatic limb fractures. Nevertheless, diagnosis of DSSI should always be combined with clinical, laboratory, and radiologic evaluations. If patients were combined with positive clinical signs, symptoms, or other laboratory tests (erythrocyte sedimentation rate and C-reactive protein), DSSI should be highly suspected when PLT $>215.50 \times 10^{9} / \mu \mathrm{L}$ and PDW $<10.35 \%$.

\section{Acknowledgment}

This work was supported by National Natural Science Foundation of China (grant no 81871762) and the Nanjing Key Program of Medical Science and Technology Development (grant nos ZKX14021 and JQX16021).

\section{Author contributions}

Zitao Zhang drafted the manuscript. Yuanhui Ji conducted the CBC analysis. Zhen Wang and Xusheng Qiu screened patients. Yixin Chen revised the article. All authors contributed to data analysis, drafting and revising the article, gave final approval of the version to be published, and agree to be accountable for all aspects of the work.

\section{Disclosure}

The authors report no conflicts of interest in this work.

\section{References}

1. Bonnevialle P. Operative treatment of early infection after internal fixation of limb fractures (exclusive of severe open fractures. Orthop Traumatol Surg Res. 2017;103(1S):S67-S73.

2. Morgenstern M, Kühl R, Eckardt H, et al. Diagnostic challenges and future perspectives in fracture-related infection. Injury. 2018;49(Suppl 1):S83-S90. 
3. Wang S, Yin P, Quan C, et al. Evaluating the Use of Serum Inflammatory Markers for Preoperative Diagnosis of Infection in Patients with Nonunions. Biomed Res Int. 2017;2017:9146317-5.

4. Celik U, Celik T, Tolunay O, et al. Platelet Indices in the Diagnosis of Ventriculoperitoneal Shunt Infection in Children. Turk Neurosurg. 2017;27(4):590-593.

5. Öztürk ZA, Dag MS, Kuyumcu ME, et al. Could platelet indices be new biomarkers for inflammatory bowel diseases? Eur Rev Med Pharmacol Sci. 2013;17(3):334-341.

6. Lee IR, Shin JI, Park SJ, Oh JY, Kim JH. Mean platelet volume in young children with urinary tract infection. Sci Rep. 2015;5:18072.

7. Dinc B, Oskay A, Dinc SE, Bas B, Tekin S. New parameter in diagnosis of acute appendicitis: platelet distribution width. World J Gastroenterol. 2015;21(6):1821-1826.

8. Ovaska MT, Mäkinen TJ, Madanat R, Vahlberg T, Hirvensalo E, Lindahl J. Predictors of poor outcomes following deep infection after internal fixation of ankle fractures. Injury. 2013;44(7):1002-1006.
9. Wang Y, Tan X, Gao H, et al. Magnitude of Soluble ST2 as a Novel Biomarker for Acute Aortic Dissection. Circulation. 2018;137(3):259-269.

10. Deppermann C, Kubes P. Platelets and infection. Semin Immunol. 2016;28(6):536-545.

11. Zhao D, Qiu G, Luo Z, Zhang Y. Platelet parameters and (1, 3)- $\beta$-Dglucan as a diagnostic and prognostic marker of invasive fungal disease in preterm infants. PLoS One. 2015;10(4):e0123907.

12. Wang F, Meng Z, Li S, Zhang Y, Wu H. Platelet Distribution Width Levels Can Be a Predictor in the Diagnosis of Persistent Organ Failure in Acute Pancreatitis. Gastroenterol Res Pract. 2017;2017:8374215-6.

13. Bath PM, Butterworth RJ. Platelet size: measurement, physiology and vascular disease. Blood Coagul Fibrinolysis. 1996;7(2):157-161.

14. Unsal E, Aksaray S, Köksal D, Sipit T. Potential role of interleukin 6 in reactive thrombocytosis and acute phase response in pulmonary tuberculosis. Postgrad Med J. 2005;81(959):604-607.
Infection and Drug Resistance

\section{Publish your work in this journal}

Infection and Drug Resistance is an international, peer-reviewed openaccess journal that focuses on the optimal treatment of infection (bacterial, fungal and viral) and the development and institution of preventive strategies to minimize the development and spread of resistance. The journal is specifically concerned with the epidemiology of antibiotic

\section{Dovepress}

resistance and the mechanisms of resistance development and diffusion in both hospitals and the community. The manuscript management system is completely online and includes a very quick and fair peerreview system, which is all easy to use. Visit http://www.dovepress.com/ testimonials.php to read real quotes from published authors. 